\section{Cancer, angiogenesis and fractals}

To the editor-The recent commentary by Coffey ${ }^{1}$ emphasizes the value of nonlinear dynamics and fractals as tools for studying aspects of biological pattern formation that are not readily accessible to strictly molecular methods. Our studies of the vascular architecture in tumors ${ }^{2-5}$ support Coffey's points about the delicate interplay among gradients in diffusible cytokines, heterogeneity of cell pheno-

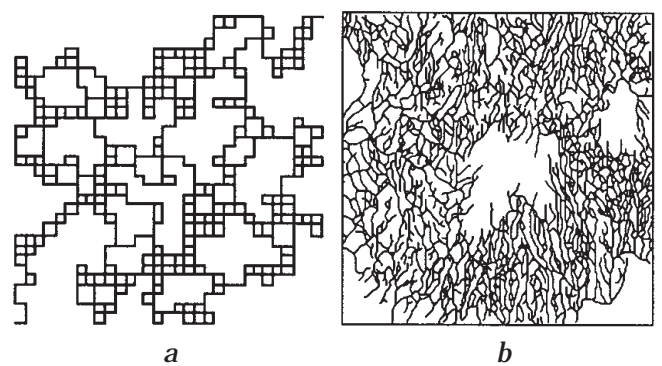

a, Computer generated percolation network and b, tumor vasculature which typically yields a fractal dimension of $1.89 \pm 0.04$.

types and cellular interactions with the extracellular matrix. In fact, by using fractal image analysis techniques, we find that tumor vascular architecture is determined by heterogeneity in the cellular interaction with the extracellular matrix rather than by gradients in diffusible angiogenic factors ${ }^{2}$. This finding has implications not only for conventional cancer therapy, but also for the antiangiogenic approaches pioneered by Judah Folkman.

Endothelial cells in tumor blood vessels respond to angiogenic factors by upregulating their rates of proliferation, migration and differentiation and lowering their rate of apoptosis, but these effects are not sufficient to explain the resultant vascular architecture. Tumors are in fact awash in angiogenic factors. As a result, gradients in growth factors are either non-existent or so completely random that an orderly vascular architecture cannot arise. In the absence of the controlled release of growth factors that might arise in normal tissue from oxygen gradients, the tumor vasculature responds by growing vigorously wherever and whenever it can through a heterogeneous extracellular matrix, a process known as invasion percolation (see figure).

Using a computer model of the tumor vasculature based on percolation processes, we investigated the transport of drugs and oxygen to tumors ${ }^{3}$. We

EMmANUEL T. RAKITZIS Surely some mistake! found that as a network deviates from the regular patterns of a normal capillary bed and behaves like a percolation network, the overall and local transport ability of the network is impaired. Percolation processes produce regions of stagnant flow and highly variable intervascular distances that leave large regions of the tumor far from the nearest blood vessel. Tumor vascular networks lack the overall network optimization that arises in normal tissues.

Our studies of the growth and subsequent regression of androgen-dependent (Shionogi) tumors $^{4,5}$ raise some interesting questions about anti-angiogenic treatment. Blood vessels in tumors regressing after hormone removal had the notable characteristics of not only reducing their overall density but also returning to fractal scaling and function similar to those of normal vessels, indicating that reducing the numbers of vessels might actually improve transport of blood-borne substances to the tumor. Although this may be desirable because traditional treatments can now be used more effectively, a tumor that previously had its growth stunted by poor nutrient transport might actually thrive in the modified environment. The lack of a definitive answer to this tradeoff points strongly to the value of an architectural and physiological perspective on tumor treatment as a supplement to the powerful molecular tools available.

JAMES W. BAISH ${ }^{1} \&$ RAKESH K. JAIN ${ }^{2}$

${ }^{1}$ D epartment of Mechanical Engineering

Bucknell University

Lewisburg, PA 17837, USA

${ }^{2}$ Edwin L. Steele Laboratory

Department of Radiation Oncology

Massachusetts General Hospital

Harvard Medical School

Boston, Massachusetts 02114, USA

1. Coffey, D.S. Self-organization, complexity and chaos: The new biology for medicine. Nature Med. 4, 882-885 (1998)

2. Gazit, Y., Berk, D.A., Leunig, M., Baxter, L.T. \& Jain, R.K. Scale-invariant behavior and vascular network formation in normal and tumor tissue. Phys. Rev. Lett. 75, 2428-2431 (1995).

3. Baish, J.W. et al. Role of tumor network architecture in nutrient and drug delivery: An invasion percolation-based network model. Microvasc. Res. 51, 327-346 (1996).

4. Gazit, $Y$ et al. Fractal characteristics of tumor vascular architecture during tumor growth and regression. Microcirculation 4, 395-402 (1997).

5. Jain, R.K. et al. Endothelial cell death, angiogenesis, and microvascular function following castration in an androgen-dependent tumor: Role of vascular endothelial growth factor. Proc. Natl. Acad. Sci. USA 95, 10820-10825 (1998).

\title{
Nattering on about names
}

To the editor-Your editorial "Molecular medicine growing pains" (Nature M ed. 4, 641; 1998) is an example of the use of a new scientific jargon. Basic research in medicine, biology, biochemistry, etc. is now to be referred to as 'molecular medicine' or 'biomedical research'. A few comments may not be amiss:

'Molecular medicine' presumably is equivalent to 'medical research at the molecular level'. However, work at the molecular level has been the constant aspiration of chemical research in general, and of medical research in particular, since the time of John Dalton, Amedeo Avogadro, Joseph Priestley and many others. Was the work of Louis Pasteur and of Paul Ehrlich anything but work at the molecular level, as known to them and their contemporaries? Again, 'biomedical' is a hybrid of 'biological' and 'medical', as if biology and medicine were alien disciplines. 'Biomedical' is a Humpty Dumpty portmanteau word. What happened to William of Ockham's splendid piece of advice: "Pluralitas non est ponenda sine necessitate (Plurality should not be posited unnecessarily)"?

"Molecular biology", a prominent American biochemist used to say, "is practicing biochemistry without a license". This, I believe, is the raison d' etre of the new scientific jargon. With a change in vocabulary, new vistas of application open. Proper training and competence can now be disregarded. All one has to do is assume a cloak of 'molecularity'. Is there a way out? Nonmolecular scientists of the world unite. You have nothing to lose but your (reaction) chains.

Department of Biological Chemistry, University of Athens M edical School, Athens 11527, Greece

Editors note: We note from his letterhead that the author is an Associate Professor of "Biochemistry" 